\title{
The exact controllability of Euler-Bernoulli beam systems with small delays in the boundary feedback controls
}

\author{
Zhang Zhuo \\ Basic Course Department, Business College of Shanxi University, Taiyuan 030031, Shanxi, P. R. China. \\ Communicated by A. Atangana
}

\begin{abstract}
This work is concerned with the exact controllability of an Euler-Bernoulli beam system with small delays in the boundary feedback controls

with boundary conditions

$$
\begin{aligned}
& w(0, t)=w_{x}(0, t)=0, \quad t \geqslant 0, \\
& w_{x x}(1, t-\varepsilon)=-k_{2}^{2} w_{t x}(1, t)-c_{2} w_{t}(1, t-\varepsilon), \quad \varepsilon>0, \quad k_{1}^{2}+k_{2}^{2} \neq 0, \\
& w_{x x x}(1, t)=k_{1}^{2} w_{t}(1, t-\varepsilon)-c_{1} w_{t x}(1, t-\varepsilon), \quad k_{i}, c_{i} \in R, \quad(i=1,2),
\end{aligned}
$$$$
w_{\mathrm{tt}}(x, \mathrm{t})+w_{x x x x}(x, t)=0, \quad x \in(0,1), \quad \mathrm{t}>0,
$$

$$
w(x, t)=\varphi(x, t), \quad w_{t}(x, t)=\psi(x, t), \quad-\varepsilon \leqslant t \leqslant 0 .
$$

Our analysis relies on the exact controllability on Hilbert space $M$ and state space $H$. Our results based on formulating the original system as a state linear system. We formulate the system as the state feedback control systems $\sum(A, B, C)$, and we get the generalized eigenvectors of the operator $A$. Then we prove that they can form a Riesz basis for the state space $H$. In the end, the system is proved to be exactly controllable on H. (C)2017 All rights reserved.
\end{abstract}

Keywords: Euler-Bernoulli beam, delay, boundary feedback control, exact controllability. 2010 MSC: 35J30, 35J35.

\section{Introduction}

Euler-Bernoulli beam systems arise in many fields such as physics, mechanics, chemistry, economics, engineering and biological science, etc., see $[1-5,10,12]$ for example. In these papers, the authors considered the exact controllability of the Euler-Bernoulli plate equation with variable coefficients and mixed boundary conditions. By using the methods of basis perturbation and Weiss regular system, the papers in [7-9] showed that the generalized eigenfunction of a flexible Euler-Bernoulli beam system with boundary feedback consisting of velocity and angular velocity form a Riesz basis for the state space $\mathrm{H}$ and they gave the computational formula exponential decay rate of the system, which solved the problems considered in engineering, such as exponential decay rate, eigenvalues and so on. But there is always time-delay in engineering practice. So the research of it is also active up recently, see $[6,11,13]$ and the

Email address: zzswxy@126.com (Zhang Zhuo)

doi:10.22436/jnsa.010.05.44 
references therein. In these papers, many authors investigated the robust stability of time-delay system with time-varying uncertainties via HPLKF. They gave a sufficient condition to demonstrate that the system is asymptotically stable. A new class of Lyapunov-Krasovskii function is introduced, whose main feature is that the conservativeness due to uncertainties is reduced. Numerical examples were given to illustrate the effectiveness of their method.

In this paper, we discuss the following flexible Euler-Bernoulli beam system with small delays in boundary feedback controls

$$
\begin{aligned}
& w_{t t}(x, t)+w_{x x x}(x, t)=0, \quad x \in(0,1), \quad t>0, \\
& w(0, t)=w_{x}(0, t)=0, \quad t \geqslant 0, \\
& w_{x x}(1, t-\varepsilon)=-k_{2}^{2} w_{t x}(1, t)-c_{2} w_{t}(1, t-\varepsilon), \varepsilon>0, \quad k_{1}^{2}+k_{2}^{2} \neq 0, \\
& w_{x x x}(1, t)=k_{1}^{2} w_{t}(1, t-\varepsilon)-c_{1} w_{t x}(1, t-\varepsilon), \quad k_{i}, c_{i} \in R, \quad(i=1,2),
\end{aligned}
$$

with boundary conditions

$$
w(x, t)=\varphi(x, t), \quad w_{t}(x, t)=\psi(x, t), \quad-\varepsilon \leqslant t \leqslant 0 .
$$

Different from the works mentioned above, we formulate the system (1.1) as the state feedback control systems $\sum(A, B, C)$, where $A$ is the generator of a $C_{0}$-semigroup on $H, B$ is a control operator and $C$ is an observation operator. Moreover, $B$ and $C$ are unbounded operators. And we formulate the system without time-delay as abstract differential functions on the Hilbert space $X$ of the form

$$
\left\{\begin{array}{l}
\frac{d \omega(t)}{d t}=A_{1} w(t), \\
\omega(0)=\left(u(x, 0), u_{t}(x, 0)\right)^{\top} .
\end{array}\right.
$$

We get the eigenvalues and eigenvectors of the operator $A$ through studying the eigenvalues and eigenvectors of the operator $A_{1}$. Then we prove that $\sum(A, B, C)$ is exactly controllable on $H$, which is generalized by the eigenvectors of the operator $A$. Since the system (1.1) and the system $\sum(A, B, C)$ are equivalent, we only need to study the exact controllability of $\sum(A, B, C)$. In the end, (1.1) is proved to be exactly controllable on $\mathrm{H}$.

\section{Riesz basis of auxiliary system}

To begin with, let

$$
\begin{aligned}
H^{(n)}(0,1)=\{\varphi:(0,1) \rightarrow & \left.C \mid \varphi^{(k)} \text { is absolutely continuous }(k=1,2, \cdots, n-1), \varphi^{(n)} \in L^{2}(0,1)\right\}, \\
& H_{0}^{2}(0,1)=\left\{\varphi \in H^{2}(0,1) \mid \varphi(0)=\varphi^{\prime}(0)=0\right\} .
\end{aligned}
$$

We denote

$$
X=\mathrm{H}_{0}^{2}(0,1) \times \mathrm{L}^{2}(0,1)
$$

where

$$
\left((f, g)^{\top},(u, v)^{\top}\right) x=\int_{0}^{1} f^{\prime \prime}(x) \overline{u^{\prime \prime}(x)} d x+\int_{0}^{1} g(x) \overline{v(x)} d x .
$$

Then, $X$ is a Hilbert space under (2.1). Let

$$
M=X \times L^{2}([-\varepsilon, 0], X),
$$

where

$$
\left((p, f(\cdot))^{\top},(q, g(\cdot))^{\top}\right)_{M}=(p, q)_{X}+\int_{-\varepsilon}^{0}(f(\theta), g(\theta))_{X} d \theta
$$


Then $M$ is a Hilbert space under (2.2). Construct the system

$$
\begin{aligned}
& w_{\mathrm{tt}}(x, \mathrm{t})+w_{x x x x}(x, \mathrm{t})=0, \quad x \in(0,1), \quad \mathrm{t}>0, \\
& w(0, \mathrm{t})=w_{x}(0, \mathrm{t})=0, \mathrm{t} \geqslant 0, \\
& w_{x x}(1, \mathrm{t})=-k_{2}^{2} w_{\mathrm{tx}}(1, \mathrm{t})-c_{2} w_{\mathrm{t}}(1, \mathrm{t}), \quad k_{1}^{2}+k_{2}^{2} \neq 0, \\
& w_{x x x}(1, \mathrm{t})=k_{1}^{2} w_{\mathrm{t}}(1, \mathrm{t})-c_{1} w_{\mathrm{tx}}(1, \mathrm{t}), \quad k_{i}, \mathrm{c}_{\mathrm{i}} \in R, \quad(i=1,2), \\
& w(x, 0)=\varphi(x, 0), \quad w_{\mathrm{t}}(x, 0)=\psi(x, 0) .
\end{aligned}
$$

Let

$$
\begin{gathered}
A_{1}=\left(\begin{array}{cc}
0 & I \\
-\frac{d^{4}}{d x^{4}} & 0
\end{array}\right), \\
D\left(A_{1}\right)=\left\{(u, v)^{\top} \in X \mid u \in H^{4}(0,1) \bigcap H_{0}^{2}(0,1), v \in H_{0}^{2}(0,1), u^{\prime \prime \prime}(1)=k_{1}^{2} v_{1}(1)-c_{1} v^{\prime}(1),\right. \\
\left.u^{\prime \prime}(1)=-k_{2}^{2} v^{\prime}(1)-c_{2} v(1)\right\} .
\end{gathered}
$$

Lemma 2.1 ([9]). Let $A_{1}$ be as in definition (2.3), then

(i) $\mu_{n k}=\tau_{n k}^{2}(k=1,2, n \geqslant 1)$ is the eigenvalue of $A_{1}$,

$$
\tau_{n k}=\rho_{n k}+o\left(\frac{1}{\rho_{n k}}\right) ;
$$

(ii) we can denote generalized eigenvectors of $A_{1}$ by $\left\{F_{0}, F_{n k} \mid k=1,2, n \geqslant 1\right\}$ (see [7]), where

$$
F_{n k}(x)=\frac{F\left(x, \tau_{n k}\right)}{\left\|G\left(x, \rho_{n k}\right)\right\|_{x}}, \quad(k=1,2, n \geqslant N)
$$

For the proof we refer the reader to [9, Theorem 3.2], where $\rho_{n k}(k=1,2), F\left(x, \tau_{n k}\right), G\left(x, \rho_{n k}\right)$ can be seen [9, Eqs. (3.3), (4.2)]. The generalized eigenvectors of $A_{1}$, i.e. $\left\{F_{0}, F_{n k} \mid k=1,2, n \geqslant 1\right\}$ form a Riesz basis for the state space $X$ (this result is contained in [8]), so $A_{1}$ is the infinitesimal generator of a $\mathrm{C}_{0}$-semigroup on the state space.

In order to formulate (1.1) as a state linear system, let $\mathrm{H}$ be a Hilbert space, $\mathrm{A}$ be the generator of a strongly continuous semigroup $T$ on $H$. For any $\mu \in \rho(A)$, we denote by $H_{-1}$ the completion of $H$ with respect to the norm $\|x\|_{-1}=\left\|\left(\mu I-A^{-1}\right) x\right\|$. This space is isomorphic to $D\left(A^{*}\right)^{*}$, and we have

$$
\mathrm{D}(\mathrm{A})=\mathrm{H}_{1} \subseteq \mathrm{H} \subseteq \mathrm{H}_{-1}=\mathrm{D}\left(\mathrm{A}^{*}\right)^{*}, \quad \mathrm{~A} \in \mathrm{L}\left(\mathrm{H}_{1}, \mathrm{H}\right) \cap \mathrm{L}\left(\mathrm{H}, \mathrm{H}_{-1}\right) .
$$

So $\widetilde{A}: H \rightarrow \mathrm{H}_{-1}$ is the extension of $\mathrm{A}$ :

$$
\left.\left(\widetilde{A}\left(\begin{array}{c}
p \\
f(\cdot)
\end{array}\right),\left(\begin{array}{c}
q \\
g(\cdot)
\end{array}\right)\right)_{D\left(A^{*}\right)^{*} \times D\left(A^{*}\right)}=(\underset{f(\cdot)}{p}), A^{*}(\underset{g(\cdot)}{q})\right)_{X},
$$

where

$$
\left(\begin{array}{c}
p \\
f(\cdot)
\end{array}\right) \in \mathrm{H},\left(\begin{array}{c}
q \\
g(\cdot)
\end{array}\right) \in \mathrm{D}\left(A^{*}\right)
$$

Hence $\widetilde{A} \in \mathcal{L}\left(H, H_{-1}\right)$. For convenience, we denote $\widetilde{A}$ by $A$ in the following. Let

Output function:

$$
\begin{aligned}
& B=\left(\begin{array}{c}
B_{0} \\
0
\end{array}\right), \quad B_{0}^{*}\left(\begin{array}{l}
f \\
g
\end{array}\right)=\left(\begin{array}{c}
-k_{2}^{-2} f_{x x}(1) \\
g(1)
\end{array}\right), \\
& C \xi(x, \cdot)=C\left(\begin{array}{c}
p(x) \\
f(x, \cdot)
\end{array}\right)=\left(\begin{array}{c}
-k_{2}^{2} f \cdot x(1, t) \\
f_{x x x}(1, t)
\end{array}\right) .
\end{aligned}
$$

Input function:

$$
y(t)=\left(\begin{array}{c}
-k_{2}^{2} w_{t x}(1, t) \\
w_{x x x}(1, t)
\end{array}\right)
$$

$$
u(t)=\left(\begin{array}{c}
w_{x x}(1, t-\varepsilon)+c_{2} w_{t}(1, t-\varepsilon) \\
k_{1}^{2} w_{t}(1, t-\varepsilon)-c_{1} w_{t x}(1, t-\varepsilon)
\end{array}\right)
$$




\section{The exact controllability of system (1.1)}

Let

$$
\begin{gathered}
A=\left(\begin{array}{cc}
A_{1} & 0 \\
0 & \frac{d}{d \theta} I
\end{array}\right), \\
D(A)=\left\{(p, f(\cdot))^{\top} \in D\left(A_{1}\right) \times H^{1}([-\varepsilon, 0], X) \mid f(0)=p\right\},
\end{gathered}
$$

where $H^{1}([-\varepsilon, 0], X)=\left\{f:[-\varepsilon, 0] \rightarrow X \mid f\right.$ is absolutely continuous on $\left.[-\varepsilon, 0], f^{\prime}(\cdot) \in L^{2}([-\varepsilon, 0], X)\right\}$. Since $\mathrm{D}(\mathrm{A}) \hookrightarrow M$ is a compact embedding, $A$ is a discrete operator.

Theorem 3.1. Let A, B, C be as in definitions (3.1), (2.4), (2.5), then system (1.1) can be formulated as the state linear systems $\sum(A, B, C)$ :

$$
\left\{\begin{array}{l}
\frac{d z(t)}{d t}=A z(t)+B u(t), \quad(t>0), \\
y(t)=C z(t), \\
z(0)=\left(\begin{array}{c}
x(0) \\
f(\cdot)
\end{array}\right),
\end{array}\right.
$$

where $z(t)=\left(\begin{array}{c}x(t) \\ x(t+\cdot)\end{array}\right), x(t)=\left(\begin{array}{c}w(\cdot, t) \\ w_{t}(\cdot, t)\end{array}\right)$, and $w(\cdot, t)$ is the solution of $(1.1)$.

Proof. Take $\left(\begin{array}{c}\mathrm{q} \\ \mathbf{g}(\cdot)\end{array}\right) \in \mathrm{D}(A)$, where $\mathrm{q}=\left(\begin{array}{c}\mathrm{q}_{1} \\ \mathrm{q}_{2}\end{array}\right) \in \mathrm{D}\left(\mathrm{A}_{1}\right), \mathrm{g}(\cdot)=\left(\begin{array}{l}\mathrm{g}_{1} \\ \mathrm{~g}_{2}\end{array}\right) \in \mathrm{D}(\mathrm{F})$, then

$$
\begin{aligned}
& \left(\frac{\mathrm{d} z(\mathrm{t})}{\mathrm{dt}}, \quad\left(\begin{array}{c}
\mathrm{q} \\
\mathrm{g}(\cdot)
\end{array}\right)\right)_{H}=\left(\frac{\mathrm{dx}(\mathrm{t})}{\mathrm{dt}}, \mathrm{q}\right)_{X}+(F x(t+\cdot), \quad g(\cdot))_{X[-\varepsilon, 0]}
\end{aligned}
$$

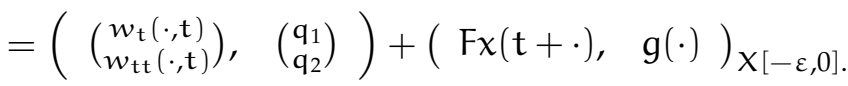

Let us prove the first equation now:

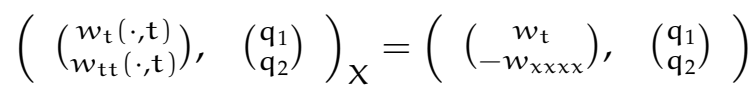

$$
\begin{aligned}
& =\int_{0}^{1} w_{\mathrm{txx}} \overline{\mathrm{q}_{1}^{\prime \prime}} \mathrm{dx}-\int_{0}^{1} w_{x x x x} \overline{\mathrm{q}_{2}} \mathrm{dx} \\
& =\left.w_{\mathrm{t} x} \overline{\mathrm{q}_{1}^{\prime \prime}}\right|_{0} ^{1}-\left.w_{\mathrm{t}} \overline{\mathrm{q}_{1}^{\prime \prime \prime}}\right|_{0} ^{1}+\int_{0}^{1} w_{\mathrm{t}} \overline{\mathrm{q}_{1}^{\prime \prime \prime \prime}} \mathrm{d} x-\left.w_{x \times x} \overline{\mathrm{q}_{2}}\right|_{0} ^{1}+\left.w_{x \times} \overline{\mathrm{q}_{2}^{\prime}}\right|_{0} ^{1}-\int_{0}^{1} w_{x \times} \overline{\mathrm{q}_{2}^{\prime \prime}} \mathrm{dx} \\
& =w_{\mathrm{tx}}(1, \mathrm{t}) \overline{\mathbf{q}_{1}^{\prime \prime}(1)}-w_{x x x}(1, \mathrm{t}) \overline{\mathbf{q}_{2}(1)}+\left(A_{0}\left(\begin{array}{c}
w(\cdot, \mathrm{t}) \\
w_{\mathrm{t}}(\cdot, \mathrm{t})
\end{array}\right), \quad\left(\begin{array}{l}
\mathbf{q}_{1} \\
\mathbf{q}_{2}
\end{array}\right)\right) \\
& =\left[-k_{2}^{-2} w_{x x}(1, t-\varepsilon)-k_{2}^{-2} c_{2} w_{t}(1, t-\varepsilon)\right] \overline{q_{1}^{\prime \prime}(1)}-\left[k_{1}^{2} w_{t}(1, t-\varepsilon)-c_{1} w_{t x}(1, t-\varepsilon)\right] \\
& \overline{\mathrm{q}_{2}(1)}+\left(A_{0}\left(\begin{array}{c}
w(\cdot, \mathrm{t}) \\
w_{\mathrm{t}}(\cdot, \mathrm{t})
\end{array}\right), \quad\left(\begin{array}{l}
\mathrm{q}_{1} \\
\mathbf{q}_{2}
\end{array}\right)\right) \\
& =\left(A_{0}\left(\begin{array}{c}
w(\cdot, t) \\
w_{t}(\cdot, t)
\end{array}\right), \quad\left(\begin{array}{l}
q_{1} \\
q_{2}
\end{array}\right)\right)+\left(u(t), \quad B_{0}^{*}\left(\begin{array}{l}
q_{1} \\
q_{2}
\end{array}\right)\right) \\
& =\left(A_{0} x(t)+B_{0} u(t)\right)\left(\begin{array}{l}
q_{1} \\
q_{2}
\end{array}\right) \text {. }
\end{aligned}
$$

Therefore,

$$
\begin{aligned}
& \left(\frac{d z(t)}{d t},\left(\begin{array}{c}
q \\
g(\cdot)
\end{array}\right)\right)_{H}=\left(A_{0} x(t)+B_{0} u(t)\right)\left(\begin{array}{l}
q_{1} \\
q_{2}
\end{array}\right)+(F x(t+\cdot), \quad g(\cdot))_{X[-\varepsilon, 0]} \\
& =\left(A_{0} x(t)+B_{0} u(t)\right) q+(F x(t+\cdot), \quad g(\cdot))_{X[-\varepsilon, 0]} \\
& =(A z(t)+B u(t))\left(\begin{array}{c}
q \\
g(\cdot)
\end{array}\right) \text {. }
\end{aligned}
$$

From the above, we can see that these families of operators constitute an alternative description of the system. 
Now we are going to consider the eigenvalue problem of the operator $A$ to research the exact controllability of system (1.1) in the following part.

Theorem 3.2. With the above notations, the following assertions hold true:

(i) if $(\lambda, r)$ is a pair of eigenvalues of $A_{1}$, then $\left.\left(\lambda, \underset{e^{\lambda} \cdot r}{r}\right)\right)$ is a pair of eigenvalues of $A$. On the other hand, if $\left(\begin{array}{c}p \\ f(\cdot)\end{array}\right)$ is the eigenvectors of $A$ corresponding to $\lambda$, then $\left(\begin{array}{c}p \\ f(\cdot)\end{array}\right)=\left(\begin{array}{c}p \\ e^{\lambda \cdot p}\end{array}\right)$;

(ii) there exists a constant $N$, when $n \geqslant N$, the eigenvalues of $A$ : $\mu_{n k},(k=1,2)$ (see Lemma 2.1$)$ are simple.

Proof.

(1) Firstly, for any $\lambda \in \sigma\left(A_{1}\right), \lambda \neq 0$, since $A_{1}$ is discrete, we know that $\lambda \in \sigma_{p}\left(A_{1}\right)$, take $r \neq 0$,

$r \in D\left(A_{1}\right)$ and $\left(\lambda I-A_{1}\right) r=0$, then $\left(\begin{array}{c}r \\ e^{\lambda \cdot r} \cdot r\end{array}\right) \in D(A)$. Further, $(\lambda I-A)\left(\begin{array}{c}r \\ e^{\lambda} \cdot r\end{array}\right)=0$. So $\sigma_{p}\left(A_{1}\right) \subset \sigma_{p}(A)$, moreover, $\left(\begin{array}{c}r \\ e^{\lambda \cdot r}\end{array}\right)$ is the eigenvectors of $A$ corresponding to the $\lambda$. Set $\lambda \neq 0, \lambda \in \sigma_{p}(A)$, take $(\underset{f}{p}(\theta)$ ) as the eigenvectors of $A$ corresponding to the $\lambda$, then $p \in D\left(A_{1}\right)$ and

$$
(\lambda I-A)\left(\begin{array}{c}
p \\
f(\theta)
\end{array}\right)=\left(\begin{array}{c}
\left(\lambda I-A_{1}\right) p \\
\lambda f(\theta)-\frac{d}{d \theta} f(\theta)
\end{array}\right)=\left(\begin{array}{l}
0 \\
0
\end{array}\right) .
$$

From $\left(\lambda I-A_{1}\right) p=0$, we obtain $\lambda \in \sigma\left(A_{1}\right)$ and $\sigma_{p}(A) \subset \sigma_{p}\left(A_{1}\right)$. So $\sigma_{p}(A)=\sigma_{p}\left(A_{1}\right)$. Solving this equation

$$
\left\{\begin{array}{l}
\frac{d f(\theta)}{d t}=\lambda f(\theta), \\
f(0)=p,
\end{array}\right.
$$

we have $f(\theta)=e^{\lambda \theta} p$.

(2) In the following, we will prove that there exists a constant $N$, when $n \geqslant N$, the eigenvalues of $A$, $\mu_{n k},(k=1,2)$ are simple.

Actually, if $(\lambda I-A)^{2}(\underset{f}{p}(\theta))=0$, denotes

$$
(\lambda I-A)\left(\begin{array}{c}
p \\
f(\cdot)
\end{array}\right)=\left(\begin{array}{c}
q \\
g(\cdot)
\end{array}\right)
$$

then $A_{1} q=\lambda q, g(\cdot)=e^{\lambda \cdot q}$. For

$$
(\lambda I-A)^{2}\left(\begin{array}{c}
p \\
f(\cdot)
\end{array}\right)=[\lambda(\lambda I-A)-A(\lambda I-A)]\left(\begin{array}{c}
p \\
f(\cdot)
\end{array}\right)=\left(\begin{array}{c}
\lambda q \\
\lambda e^{\lambda \cdot q}
\end{array}\right)-A\left(\begin{array}{c}
q \\
e^{\lambda \cdot q}
\end{array}\right)=\left(\begin{array}{c}
\lambda q \\
\lambda e^{\lambda \cdot} \cdot q
\end{array}\right)=\left(\begin{array}{l}
0 \\
0
\end{array}\right),
$$

we have $\lambda q=0, \lambda \neq 0$. It is easy to know that $q=0$. So

$$
(\lambda I-A)\left(\begin{array}{c}
p \\
f(\cdot)
\end{array}\right)=\left(\begin{array}{l}
0 \\
0
\end{array}\right), \quad \operatorname{dim}((\lambda I-A) M)=1 .
$$

Noticing $\mu_{n k}, k=1,2, n \geqslant N$ is the simple eigenvalues of $A_{1}$ (see [9, Theorem 3.2]). Evidently, it is also a simple eigenvalue of $A$.

It follows from the above theorem and Lemma 2.1 that generalized eigenvectors of $A$ are:

$$
\left\{\Phi_{n k}=\left(\begin{array}{c}
F_{n k} \\
e^{\mu_{n k} \cdot F_{n k}}
\end{array}\right) \mid k=1,2, n \geqslant N\right\}
$$

Because $A$ is discrete, all of the generalized eigenvectors can be set as

$$
\left\{\Phi_{0}, \Phi_{n k} \mid k=1,2, n \geqslant 1\right\} .
$$

Evidently, (3.3) is not complete on M. Let

$$
\mathrm{H}=\overline{\operatorname{span}}\left\{\Phi_{0}, \Phi_{n k} \mid \mathrm{k}=1,2, \mathrm{n} \geqslant 1\right\} .
$$

It is easy to see $H \neq M$ and $H$ is the $A$-invariant subspace of $M$. Using that $H$ is closed, we know $H$ is a Hilbert space with the inner product (2.2). 
For the sake of convenience, we denote by $A$ the $\left.A\right|_{H}$. So we have the following conclusions.

Lemma $3.3([8]) .\left\{\Phi_{0}, \Phi_{n k} \mid k=1,2, n \geqslant 1\right\}$ forms a Riesz basis for the state space $\mathrm{H}$. When $\mathrm{t} \geqslant 0$, let $z(t)=\left(\begin{array}{c}x(t) \\ x(t+\cdot)\end{array}\right), x(t)=\left(\begin{array}{c}w(\cdot, t) \\ w_{t}(\cdot, t)\end{array}\right)$, where $w(\cdot, t)$ is the solution of system (1.1). Denote

$$
\chi(s)=f(\cdot, s)=\left(\begin{array}{c}
\varphi(\cdot, s) \\
\psi(\cdot, s)
\end{array}\right) \quad(-\varepsilon \leqslant s \leqslant 0),
$$

then (1.1) can be rewritten as an abstract equation

$$
\left\{\begin{array}{l}
\frac{d z(t)}{d t}=A z(t), \quad t \geqslant 0 \\
z(0)=(x(0), f(\cdot))^{\top} \in H .
\end{array}\right.
$$

This implies that $A$ is the infinitesimal generator of the $C_{0}$-semigroup $\{T(t) \mid t \geqslant 0\}$. So the following results hold true.

Theorem 3.4 ([8]). Let $\mathrm{A}$ be given as in (3.1) and assume that $\{\mathrm{T}(\mathrm{t}) \mid \mathrm{t} \geqslant 0\}$ is the $\mathrm{C}_{0}$-semigroup, then we have the following conclusions:

(i) A is a Riesz spectral operator on $\mathrm{H}$;

(ii) $A \xi=\sum_{n=N}^{+\infty} \sum_{k=1,2} \mu_{n k}\left(\xi, \Phi_{n k}\right)_{H} \Psi_{n k}, \forall \xi \in H$;

(iii) $\mathrm{T}(\mathrm{t}) \xi=e^{\mu_{0} \mathrm{t}}\left(\xi, \Psi_{0}\right)_{H} \Phi_{0}+\Sigma_{n=N}^{+\infty} \sum_{k=1,2} e^{\mu_{n k} \mathrm{t}}\left(\xi, \Phi_{n k}\right)_{H} \Psi_{n k}, \forall \xi \in \mathrm{H}$, where $\mu_{0}$ is the eigenvalue of $A$ for $\Phi_{0},\left\{\Psi_{0}, \Psi_{n k} \mid k=1,2, n \geqslant 1\right\}$ is the biorthogonal column of $\left\{\Phi_{0}, \Phi_{n k} \mid k=1,2, n \geqslant 1\right\}$.

We are going to give the exact controllability of the system (3.2) in the space $H$, which is the main result of this paper.

Theorem 3.5. Let B be given as in definition (2.4), then B is an admissible control operator for $\mathrm{H}$.

Proof. We only need to prove that for any step function $u(\tau) \in \mathrm{L}^{2}([0, t])$, there exist $t>0$ and $M>0$ such that

$$
\left\|\int_{0}^{t} \mathrm{~T}(\mathrm{t}-\tau) \operatorname{Bu}(\tau) \mathrm{d} \tau\right\|_{\mathrm{H}} \leqslant M\|u\|_{\mathrm{L}^{2}([0, \mathrm{t}], \mathrm{u})}
$$

holds true. Actually,

$$
\begin{aligned}
\left\|\int_{0}^{t} T(t-\tau) B u(\tau) d \tau\right\|_{H} & =\left\|\int_{0}^{t} \sum_{k=1}^{2} \sum_{n \in N} e^{\lambda_{n k}(t-\tau)}\left(B u(\tau), \phi_{n k}\right) u \psi_{n k} d \tau\right\|_{H} \\
& =\left\|\int_{0}^{t} \sum_{k=1}^{2} \sum_{n \in N} e^{\lambda_{n k}(t-\tau)}\left(u(\tau), B^{*} \phi_{n k}\right) u \psi_{n k} d \tau\right\|_{H} \\
& \left.\left.=\| \int_{0}^{t} \sum_{k=1}^{2} \sum_{n \in N} e^{\lambda_{n k}(t-\tau)}\left(\begin{array}{c}
u_{1}(\tau) \\
u_{2}(\tau)
\end{array}\right), \underset{\varphi}{-k_{2}^{-2} \mu_{n k}^{-1} \varphi^{\prime \prime}\left(1, \tau_{n k}\right)}\right)\right)_{\varphi} \psi_{n k} d \tau \|_{H} \\
& \leqslant\left\{\sum_{k=1}^{2} \sum_{n \in N}\left|\int_{0}^{t} e^{\lambda_{n k}(t-\tau)}\left[-k_{2}^{-2} \mu_{n k}^{-1} u_{1}(\tau) \overline{\varphi^{\prime \prime}\left(1, \tau_{n k}\right)}+u_{2}(\tau) \overline{\varphi\left(1, \tau_{n k}\right)}\right] d \tau\right|^{2}\right\}^{\frac{1}{2}} \\
& \leqslant\left\{\sum _ { k = 1 } ^ { 2 } \sum _ { n \in N } \left[\left|-k_{2}^{-2} \mu_{n k}^{-1} \varphi^{\prime \prime}\left(1, \tau_{n k}\right)\left\|e^{\lambda_{n k} t}\right\| \int_{0}^{t} e^{-\lambda_{n k} \tau} u_{1}(\tau) d \tau\right|+\left|\varphi\left(1, \tau_{n k}\right) \| e^{\lambda_{n k} t}\right|\right.\right. \\
& \left.\times\left|\int_{0}^{t} e^{-\lambda_{n k} \tau} u_{2}(\tau) d \tau\right|\right]^{2}
\end{aligned}
$$


Set $u_{1}(\tau)=\sum_{i=1}^{m} c_{i} \chi_{\left[x_{i-1}, x_{i}\right]}(\tau)$, then

$$
\begin{aligned}
\left|\int_{0}^{t} e^{-\lambda_{n k} \tau} u_{1}(\tau) d \tau\right| & =\left|\sum_{i=1}^{m} c_{i} \int_{x_{i-1}}^{x_{i}} e^{-\lambda_{n k} \tau} d \tau\right| \\
& =\left|\sum_{i=1}^{m} \frac{c_{i}}{-\lambda_{n k}}\left(e^{-\lambda_{n k} x_{i}}-e^{-\lambda_{n k} x_{i-1}}\right)\right| \\
& \leqslant \sum_{i=1}^{m} \frac{\left|c_{i}\right|}{\left|\lambda_{n k}\right|} M_{1} \\
& =\frac{M_{1}}{\left|\lambda_{n k}\right|} \int_{0}^{t}\left|u_{1}(\tau)\right| d \tau .
\end{aligned}
$$

Therefore,

$$
\left|\int_{0}^{t} e^{-\lambda_{n k} \tau} u_{2}(\tau) d \tau\right| \leqslant \frac{M_{1}}{\left|\lambda_{n k}\right|} \int_{0}^{t}\left|u_{2}(\tau)\right| d \tau
$$

which is the same as the previous proof. Where

$$
\begin{aligned}
& M_{1}=\left|e^{-\lambda_{n k} x_{i}}-e^{-\lambda_{n k}} x_{i-1}\right|, \\
& M_{2}=\max \left\{M_{1},\left|-k_{2}^{-2} \mu_{n k}^{-1} \varphi^{\prime \prime}\left(1, \tau_{n k}\right)\right|,\left|e^{\lambda_{n k} t}\right|,\left|\varphi\left(1, \tau_{n k}\right)\right|\right\} .
\end{aligned}
$$

Then

$$
\begin{aligned}
\left\|\int_{0}^{t} T(t-\tau) B u(\tau) d \tau\right\|_{H} & \leqslant\left\{\sum_{k=1}^{2} \sum_{n \in N} \frac{M_{2}^{4}}{\left|\lambda_{n k}\right|^{2}}\left[\int_{0}^{t}\left|u_{1}(\tau)\right| d \tau+\int_{0}^{t}\left|u_{2}(\tau)\right| d \tau\right]^{2}\right\}^{\frac{1}{2}} \\
& \leqslant M_{3}\left[\int_{0}^{t}\left|u_{1}(\tau)\right| d \tau+\int_{0}^{t}\left|u_{2}(\tau)\right| d \tau\right]
\end{aligned}
$$

where $M_{3}=\left[\sum_{k=1}^{2} \sum_{n \in N} \frac{M_{2}^{4}}{\left|\lambda_{n k}\right|^{2}}\right]^{\frac{1}{2}}$. Furthermore,

$$
\begin{aligned}
\|u\|_{\mathrm{L}^{2}([0, \mathrm{t}], \mathrm{u})} & =\left(\int_{0}^{\mathrm{t}}\|\mathrm{u}(\tau)\|_{\mathrm{u}}^{2} \mathrm{~d} \tau\right)^{\frac{1}{2}} \\
& =\left(\int_{0}^{\mathrm{t}}\left|\mathrm{u}_{1}(\tau)\right|^{2}+\left|\mathrm{u}_{2}(\tau)\right|^{2} \mathrm{~d} \tau\right)^{\frac{1}{2}} \\
& \geqslant\left[\left(\int_{0}^{\mathrm{t}}\left|\mathrm{u}_{1}(\tau)\right| \mathrm{d} \tau\right)^{2}+\left(\int_{0}^{\mathrm{t}}\left|\mathrm{u}_{2}(\tau)\right| \mathrm{d} \tau\right)^{2}\right]^{\frac{1}{2}} \\
& \geqslant \frac{\sqrt{2}}{2}\left[\int_{0}^{\mathrm{t}}\left(\left|\mathrm{u}_{1}(\tau)\right|+\left|\mathrm{u}_{2}(\tau)\right|\right) \mathrm{d} \tau\right] .
\end{aligned}
$$

Let $M=\sqrt{2} M_{3}$, we have

$$
\left\|\int_{0}^{t} \mathrm{~T}(\mathrm{t}-\tau) \mathrm{Bu}(\tau) \mathrm{d} \tau\right\|_{\mathrm{H}} \leqslant M\|u\|_{\mathrm{L}^{2}([0, \mathrm{t}], \mathrm{u})} .
$$

Theorem 3.6. Let $\mathrm{A}, \mathrm{B}, \mathrm{C}$ and $\mathrm{T}(\mathrm{t})$ be given as in definitions (3.1), (2.4), (2.5) and Theorem 3.4 (iii), set $z=\left(\begin{array}{c}p(x) \\ f(x, \cdot)\end{array}\right)$, where $p(x)=\left(\begin{array}{l}p_{1}(x) \\ p_{2}(x)\end{array}\right), f(x, \cdot)=\left(\begin{array}{l}f_{1}(x, \cdot) \\ f_{2}(x, \cdot)\end{array}\right)$, then system (3.2) is exact controllable on $[0, \varepsilon]$.

Proof. In order to prove that system (3.2) is exactly controllable on $[0, \varepsilon]$, we only need to prove for any $\varepsilon>0$, there exists $\gamma>0$ such that $\int_{0}^{\varepsilon}\left\|\mathrm{B}^{*} \mathrm{~T}^{*}(\mathrm{t}) z\right\|_{\mathrm{U}}^{2} \mathrm{dt} \geqslant \gamma\|z\|_{\mathrm{H}}^{2}$ holds true. If we give an equivalent norm, 
$\left\{\phi_{n k} \mid k=1,2, n \in N\right\}$ will become an orthonormal basis of $H$. So, let us assume that $\left\{\phi_{n k} \mid k=1,2, n \in N\right\}$ are orthogonal for writing convenience. Actually,

$$
\begin{aligned}
& \|z\|_{\mathrm{H}}^{2}=\left\|\sum_{k=1}^{2} \sum_{n \in N}\left(z, \phi_{n k}\right)_{H} \phi_{n k}\right\|_{H}^{2}
\end{aligned}
$$

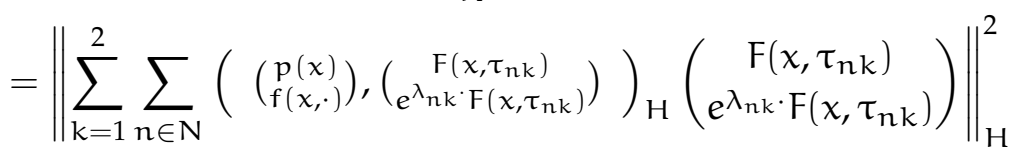

$$
\begin{aligned}
& =\sum_{k=1}^{2} \sum_{n \in N}\left|\left(\left(\begin{array}{c}
p(x) \\
f(x, \cdot)
\end{array}\right),\left(\begin{array}{c}
F\left(x, \tau_{n k}\right) \\
e^{\lambda_{n k} \cdot F\left(x, \tau_{n k}\right)}
\end{array}\right)\right)_{H}\right|^{2} \\
& \leqslant M \sum_{k=1}^{2} \sum_{n \in N} \mid \int_{0}^{1}\left[p_{1}^{\prime \prime}(x) \overline{\lambda_{n k}^{-1} \varphi^{\prime \prime}\left(x, \tau_{n k}\right)}+p_{2}(x) \overline{\varphi\left(x, \tau_{n k}\right)}\right] d x+e^{\overline{\lambda_{n k}} t} \int_{-\varepsilon}^{0} \int_{0}^{1}\left[f_{1 x x}(x, \theta)\right. \\
& \left.\times \overline{\lambda_{n k}^{-1} \varphi^{\prime \prime}\left(x, \tau_{n k}\right)}+f_{2}(x, \theta) \overline{\varphi\left(x, \tau_{n k}\right)}\right]\left.d x d \theta\right|^{2} \\
& =M \sum_{k=1}^{2} \sum_{n \in N}\left[\left(\int_{0}^{1}\left[p_{1}^{\prime \prime}(x) \overline{\lambda_{n k}^{-1} \varphi^{\prime \prime}\left(x, \tau_{n k}\right)}+p_{2}(x) \overline{\varphi\left(x, \tau_{n k}\right)}\right] d x\right)^{2}+2 e^{R e \lambda_{n k} t}\right. \\
& \times \int_{0}^{1}\left[p_{1}^{\prime \prime}(x) \overline{\lambda_{n k}^{-1} \varphi^{\prime \prime}\left(x, \tau_{n k}\right)}+p_{2}(x) \overline{\varphi\left(x, \tau_{n k}\right)}\right] d x \int_{-\varepsilon}^{0} \int_{0}^{1}\left[f_{1 x x}(x, \theta) \overline{\lambda_{n k}^{-1} \varphi^{\prime \prime}\left(x, \tau_{n k}\right)}+f_{2}(x, \theta)\right. \\
& \left.\left.\times \overline{\varphi\left(x, \tau_{n k}\right)}\right] \mathrm{d} x \mathrm{~d} \theta+\mathrm{e}^{2 \operatorname{Re} \lambda_{\mathrm{nk}} \mathrm{t}}\left(\int_{-\varepsilon}^{0} \int_{0}^{1}\left[f_{1 x x}(x, \theta) \overline{\lambda_{n k}^{-1} \varphi^{\prime \prime}\left(x, \tau_{\mathrm{nk}}\right)}+f_{2}(x, \theta) \overline{\varphi\left(x, \tau_{n k}\right)}\right] \mathrm{d} x \mathrm{~d} \theta\right)^{2}\right] .
\end{aligned}
$$

Moreover,

$$
\begin{aligned}
& \int_{0}^{\varepsilon}\left\|B^{*} T^{*}(t) z\right\|_{u}^{2} d t=\int_{0}^{\varepsilon}\left\|B^{*}\left(\sum_{k=1}^{2} \sum_{n \in N} e^{\overline{\lambda_{n k}} t}\left(z, \phi_{n k}\right)_{H} \phi_{n k}\right)\right\|_{u}^{2} d t \\
& \left.=\int_{0}^{\varepsilon} \| \sum_{k=1}^{2} \sum_{n \in N} e^{\overline{\lambda_{n k}} t}\left(\begin{array}{c}
p(x) \\
f(x, \cdot)
\end{array}\right),\left(\begin{array}{c}
F\left(x, \tau_{n k}\right) \\
e^{\lambda} n k t F\left(x, \tau_{n k}\right)
\end{array}\right)\right)_{H}\left(\begin{array}{c}
-k_{2}^{-2} \lambda_{n k}^{-1} \varphi^{\prime \prime}\left(\tau_{n k}\right) \\
\varphi\left(1, \tau_{n k}\right)
\end{array}\right) \|_{U}^{2} d t \\
& =\int_{0}^{\varepsilon} \sum_{k=1}^{2} \sum_{n \in N}\left|e^{2 \overline{\lambda_{n k}} t}\right| \mid \int_{0}^{1}\left[p_{1}^{\prime \prime}(x) \overline{\lambda_{n k}^{-1} \varphi^{\prime \prime}\left(x, \tau_{n k}\right)}+p_{2}(x) \overline{\varphi\left(x, \tau_{n k}\right)}\right] d x \\
& +e^{\overline{\lambda_{n k}} t} \int_{-\varepsilon}^{0} \int_{0}^{1}\left[f_{1 x x}(x, \theta) \overline{\lambda_{n k}^{-1} \varphi^{\prime \prime}\left(x, \tau_{n k}\right)}\right. \\
& \left.+f_{2}(x, \theta) \overline{\varphi\left(x, \tau_{n k}\right)}\right]\left.d x d \theta\right|^{2}\left[k_{2}^{-4} \lambda_{n k}^{-2}\left|\varphi^{\prime \prime}\left(1, \tau_{n k}\right)\right|^{2}+\left|\varphi\left(1, \tau_{n k}\right)\right|^{2}\right] d t \\
& \geqslant m \int_{0}^{\varepsilon} \sum_{k=1}^{2} \sum_{n \in N} e^{2 R e \lambda_{n k} t}\left(\left(\int_{0}^{1}\left[p_{1}^{\prime \prime}(x) \overline{\lambda_{n k}^{-1} \varphi^{\prime \prime}\left(x, \tau_{n k}\right)}+p_{2}(x) \overline{\varphi\left(x, \tau_{n k}\right)}\right] d x\right)^{2}+2 e^{R e \lambda_{n k} t}\right. \\
& \times\left(\int_{0}^{1}\left[p_{1}^{\prime \prime}(x) \overline{\lambda_{n k}^{-1} \varphi^{\prime \prime}\left(x, \tau_{n k}\right)}+p_{2}(x) \overline{\varphi\left(x, \tau_{n k}\right)}\right] d x\right)\left(\int _ { - \varepsilon } ^ { 0 } \int _ { 0 } ^ { 1 } \left[f_{1 x x}(x, \theta) \overline{\lambda_{n k}^{-1} \varphi^{\prime \prime}\left(x, \tau_{n k}\right)}\right.\right. \\
& \left.\left.+f_{2}(x, \theta) \overline{\varphi\left(x, \tau_{n k}\right)}\right] d x d \theta\right)+e^{2 R e \lambda_{n k} t}\left(\int _ { - \varepsilon } ^ { 0 } \int _ { 0 } ^ { 1 } \left[f_{1 x x}(x, \theta) \overline{\lambda_{n k}^{-1} \varphi^{\prime \prime}\left(x, \tau_{n k}\right)}+f_{2}(x, \theta)\right.\right.
\end{aligned}
$$




$$
\begin{aligned}
& \left.\left.\left.\times \overline{\varphi\left(x, \tau_{n k}\right)}\right] d x d \theta\right)^{2}\right) d t \\
& =m \sum_{k=1}^{2} \sum_{n \in N}\left[\frac{1}{2 \operatorname{Re} \lambda_{n k}}\left(e^{2 R e \lambda_{n k} \varepsilon}-1\right)\left(\int_{0}^{1}\left[p_{1}^{\prime \prime}(x) \overline{\lambda_{n k}^{-1} \varphi^{\prime \prime}\left(x, \tau_{n k}\right)}+p_{2}(x) \overline{\varphi\left(x, \tau_{n k}\right)}\right] d x\right)^{2}\right. \\
& +\frac{2}{3 \operatorname{Re} \lambda_{n k}}\left(e^{3 \operatorname{Re} \lambda_{n k} \varepsilon}-1\right)\left(\int_{0}^{1}\left[p_{1}^{\prime \prime}(x) \overline{\lambda_{n k}^{-1} \varphi^{\prime \prime}\left(x, \tau_{n k}\right)}+p_{2}(x) \overline{\varphi\left(x, \tau_{n k}\right)}\right] d x\right) \\
& \times\left(\int _ { - \varepsilon } ^ { 0 } \int _ { 0 } ^ { 1 } \left[f_{1 x x}(x, \theta) \overline{\lambda_{n k}^{-1} \varphi^{\prime \prime}\left(x, \tau_{n k}\right)}\right.\right. \\
& \left.\left.+f_{2}(x, \theta) \overline{\varphi\left(x, \tau_{n k}\right)}\right] d x d \theta\right)+\frac{1}{4 \operatorname{Re} \lambda_{n k}}\left(e^{4 \operatorname{Re} \lambda_{n k} \varepsilon}-1\right)\left(\int _ { - \varepsilon } ^ { 0 } \int _ { 0 } ^ { 1 } \left[f_{1 x x}(x, \theta)\right.\right. \\
& \left.\left.\left.\times \overline{\lambda_{n k}^{-1} \varphi^{\prime \prime}\left(x, \tau_{n k}\right)}+f_{2}(x, \theta) \overline{\varphi\left(x, \tau_{n k}\right)}\right] d x d \theta\right)^{2}\right] \\
& \geqslant m \min \left\{\frac{1}{2 \operatorname{Re} \lambda_{n k}}\left(e^{2 \operatorname{Re} \lambda_{n k} \varepsilon}-1\right), \frac{2}{3 \operatorname{Re} \lambda_{n k}}\left(e^{3 \operatorname{Re} \lambda_{n k} \varepsilon}-1\right), \frac{1}{4 \operatorname{Re} \lambda_{n k}}\left(e^{4 \operatorname{Re} \lambda_{n k} \varepsilon}-1\right), 1,\right. \\
& \left.2 e^{R e \lambda_{n k} t}, e^{2 \operatorname{Re} \lambda_{n k} t}\right\}\left(\left(\int_{0}^{1}\left[p_{1}^{\prime \prime}(x) \overline{\lambda_{n k}^{-1} \varphi^{\prime \prime}\left(x, \tau_{n k}\right)}+p_{2}(x) \overline{\varphi\left(x, \tau_{n k}\right)}\right] d x\right)^{2}+2 e^{R e \lambda_{n k} t}\right) \\
& \times\left(\int_{0}^{1}\left[p_{1}^{\prime \prime}(x) \overline{\lambda_{n k}^{-1} \varphi^{\prime \prime}\left(x, \tau_{n k}\right)}+p_{2}(x) \overline{\varphi\left(x, \tau_{n k}\right)}\right] d x\right)\left(\int _ { - \varepsilon } ^ { 0 } \int _ { 0 } ^ { 1 } \left[f_{1 x x}(x, \theta) \overline{\lambda_{n k}^{-1} \varphi^{\prime \prime}\left(x, \tau_{n k}\right)}\right.\right. \\
& \left.\left.+f_{2}(x, \theta) \overline{\varphi\left(x, \tau_{n k}\right)}\right] d x d \theta\right)+e^{2 R e \lambda_{n k} t}\left(\int _ { - \varepsilon } ^ { 0 } \int _ { 0 } ^ { 1 } \left[f_{1 x x}(x, \theta) \overline{\lambda_{n k}^{-1} \varphi^{\prime \prime}\left(x, \tau_{n k}\right)}\right.\right. \\
& \left.\left.\left.+f_{2}(x, \theta) \overline{\varphi\left(x, \tau_{n k}\right)}\right] d x d \theta\right)^{2}\right) \\
& \geqslant \frac{m}{M} \min \left\{\frac{1}{2 \operatorname{Re} \lambda_{n k}}\left(e^{2 \operatorname{Re} \lambda_{n k} \varepsilon}-1\right), \frac{2}{3 \operatorname{Re} \lambda_{n k}}\left(e^{3 \operatorname{Re} \lambda_{n k} \varepsilon}-1\right), \frac{1}{4 \operatorname{Re} \lambda_{n k}}\left(e^{4 \operatorname{Re} \lambda_{n k} \varepsilon}-1\right), 1,\right. \\
& \left.2 e^{\operatorname{Re} \lambda_{n k} t}, e^{2 \operatorname{Re} \lambda_{n k} t}\right\}\|z\|_{H}^{2},
\end{aligned}
$$

where

$$
m=\inf _{k=1,2, n \in N}\left[k_{2}^{-4} \lambda_{n k}^{-2}\left|\varphi^{\prime \prime}\left(1, \tau_{n k}\right)\right|^{2}+\left|\varphi\left(1, \tau_{n k}\right)\right|^{2}\right]
$$

Take

$$
\gamma=\frac{m}{M} \min \left\{\frac{1}{2 \operatorname{Re} \lambda_{n k}}\left(e^{2 \operatorname{Re} \lambda_{n k} \varepsilon}-1\right), \frac{2}{3 \operatorname{Re} \lambda_{n k}}\left(e^{3 \operatorname{Re} \lambda_{n k} \varepsilon}-1\right), \frac{1}{4 \operatorname{Re} \lambda_{n k}}\left(e^{4 \operatorname{Re} \lambda_{n k} \varepsilon}-1\right), 1,2 e^{\operatorname{Re} \lambda_{n k} t}, e^{2 \operatorname{Re} \lambda_{n k} t}\right\} .
$$

Therefore,

$$
\int_{0}^{\varepsilon}\left\|\mathrm{B}^{*} \mathrm{~T}^{*}(\mathrm{t}) z\right\|_{\mathrm{u}}^{2} \mathrm{dt} \geqslant \gamma\|z\|_{\mathrm{H}}^{2}
$$

Since systems (1.1) and (3.2) are equivalent, we get the exact controllability of system (1.1).

\section{Conclusion}

In this paper we discuss the flexible Euler-Bernoulli beam system with small delays in boundary feedback controls (1.1) with boundary conditions. Different from the works in literature, we formulate 
the system (1.1) as the state feedback control systems $\sum(A, B, C)$. And then we formulate the system without time-delay as abstract differential functions on the Hilbert space $X$. We get the eigenvalues and eigenvectors of the operator $A$ through studying the eigenvalues and eigenvectors of the operator $A_{1}$. Then we prove that $\sum(A, B, C)$ is exactly controllable on $H$, which is generalized by the eigenvectors of the operator $A$. Since the system (1.1) and the system $\sum(A, B, C)$ are equivalent, we only need to study the exact controllability of $\sum(A, B, C)$. In the end, (1.1) is proved to be exactly controllable on $H$.

\section{Acknowledgment}

The paper was supported by the Science Foundation of Shanxi Province (2013011003-3).

\section{References}

[1] Z.-Q. Ge, G.-T. Zhu, D.-X. Feng, Exact controllability for singular distributed parameter system in Hilbert space, Sci. China Ser. F, 52 (2009), 2045-2052. 1

[2] G. C. Gorain, S. K. Bose, Exact controllability and boundary stabilization of flexural vibrations of an internally damped flexible space structure, Appl. Math. Comput., 126 (2002), 341-360.

[3] L. Hu, F.-Q. Ji, K. Wang, Exact boundary controllability and exact boundary observability for a coupled system of quasilinear wave equations, Chin. Ann. Math. Ser. B, 34 (2013), 479-490.

[4] K. Huang, Y.-J. Yin, F. Yang, Q.-S. Fan, A modified couple stress nonlinear theory for Bernoulli-Euler microbeam, 13th International Conference on Fracture (ICF13), Abstract Book, Beijing, (2013).

[5] Y. Lü, Exact controllability for a class of nonlinear evolution control systems, Commun. Math. Res., 8 (2015), 285-288. 1

[6] G.-C. Pang, K.-J. Zhang, Stability of time-delay system with time-varying uncertainties via homogeneous polynomial Lyapunov-Krasovskii functions, Inter. J. Autom. Comput., 12 (2015), 657-663. 1

[7] R. Rebarber, Conditions for the equivalence of internal and external stability for distributed parameter systems, Proceedings of the 30th IEEE Conference on Decision and Control, Brighton, UK, 3 (1991), 3008-3013. 1, 2.1

[8] M. Y. Robert, An introduction to nonharmonic Fourier series, Revised first edition, Academic Press, Inc., San Diego, CA, (2001). 2, 3.3, 3.4

[9] Y.-T. Wang, G. Wang, S.-J. Li, On Riesz basis of Euler-Bernoulli beam system by boundary feedback controls, Acta Math. Sin. Chin. Ser., 2 (2000), 111-122. 1, 2.1, 2, 3

[10] L. Xu, S.-P. Shen, Size-dependent behavior in nano-dielectric Bernoulli-Euler beam, Abstract Book of 23rd International Congress of Theoretical and Applied Mechanics, (2012). 1

[11] R.-M. Yang, Y.-Z. Wang, Stability for a class of nonlinear time-delay systems via Hamiltonian functional method, Sci. China Inf. Sci., 55 (2012), 1218-1228. 1

[12] F.-Y. Yang, P.-F. Yao, Exact controllability of the Euler-Bernoulli plate with variable coefficients and mixed boundary conditions, 34th Chinese Control Conference (CCC), Hangzhou, China, (2015), 1395-1400. 1

[13] Y. Zhu, Q.-N. Gao, Y. Xiao, Sufficient conditions for stability of linear time-delay systems with dependent delays, J. Syst. Eng. Electron., 24 (2013), 845-851. 1 